\title{
Hypoxic microenvironment promotes proliferation and invasion of non-small cell lung cancer A549 Cells through Wnt/B-catenin signaling pathway.
}

\author{
Ruidong Ma, Xiaoping He, Hong Wang, Weikun Jia, Xiaofei Zeng* \\ Department of Cardiothoracic Surgery, the First Affiliated Hospital of Chengdu Medical College, Chengdu Sichuan, \\ PR China
}

\begin{abstract}
Objective: To study the effect of Wnt// $/$-catenin signaling pathway on hypoxia induced proliferation and invasion in lung cancer cell line A549.

Methods: Cultured lung cancer A549 cells were treated with hypoxia for $24 \mathrm{~h}$. The proliferation rates were detected by CCK8; the mRNA and protein expression levels of HIF- $1 \alpha$ and $\beta$-catenin were detected by RT-PCR and Western blot; the effect of hypoxia on invasion ability of A549 cells were detected with Transwell method. Furthermore, $\beta$-catenin siRNA was used to investigate the role of $\beta$-catenin on hypoxia induced proliferation and invasion.

Results: The results demonstrated that hypoxia could promote A549 cells proliferation and invasion. The mRNA and protein level of HIF-1 $\alpha$ and $\beta$-catenin was upregulated by hypoxia treatment, whereas $\beta$ catenin siRNA could block these processes.

Conclusion: Hypoxia promotes osteosarcoma cell line A549 proliferation and invasion by activating Wnt/ $\beta$-catenin signaling pathway.
\end{abstract}

Keywords: Lung cancer, Hypoxia, Wnt/ $\beta$-catenin, Proliferation, Invasion.

\section{Introduction}

Lung cancer is the most common malignant tumor, and frequently seen in China. According to the World Cancer Report 2014 issued by the World Health Organization (WHO), the incidence rate and mortality rate of lung cancer have boosted in the world, and rank first in all malignant tumors [1]. Among all types of lung cancers, Non-Small Cell Lung Cancer (NSCLC) occupies almost $80 \%$, but the 5 y survival rate is only $13 \%[2,3]$. Besides, NSCLC is also the most common form of lung cancer with the highest mortality rate due to its poor prognosis in spite of the promotion and application of neoadjuvant therapy and individualized comprehensive therapy $[4,5]$. Abnormal proliferation and distant metastasis have been considered as the major factors leading to NSCLC-related death, which renders a great significance to the in-depth research into the molecular mechanism for ameliorating the clinical treatment and prognosis of patient with lung cancer. During the rapid growth of solid tumors, contradictions lie between the rapid proliferation and retarded peripheral vascular growth of tumor cells in a short time, which could result in insufficient nutrition and oxygen for tumor cells, thereby generating an ischemic and hypoxic microenvironment in focal tissues of tumor [6-8]. Furthermore, hypoxic microenvironment will affect cell proliferation, angiogenesis, invasion and metastasis of tumors [9-11]. Studies have shown that $\mathrm{Wnt} / \beta$-catenin signaling pathway is involved in the proliferation, differentiation, metastasis and invasion of tumor cells $[12,13]$, but no literature has reported the effect of $\mathrm{Wnt} / \beta$ catenin signaling pathway on the proliferation, invasion and metastasis of NSCLC A549 cells under the hypoxic environment. Thus, in this study, we aimed to investigate the potential mechanism of $\mathrm{Wnt} / \beta$-catenin signaling pathway through discovering the effects of $\mathrm{Wnt} / \beta$-catenin signaling pathway on NSCLC A549 cell proliferation and invasion under the hypoxic microenvironment in an in-vitro experiment.

\section{Materials and Methods}

\section{Materials}

Human lung cancer A549 cell lines (Institute of Biochemistry and Cell Biology, SIBS, CAS); TRIzol RNA extraction kit (Invtirogen, USA); primers of HIF- $1 \alpha$ and $\beta$-catenin were designed and synthesized by Beijing Tianyi Huiyuan Biotech Co., Ltd; rabbit anti-human polyclonal antibodies of HIF-1 $\alpha$ and $\beta$-catenin (Santa Cruz, US); rabbit anti-human monoclonal antibody of $\beta$-actin (CST, US); horseradish peroxidase labeled goat anti-rabbit IgG (Boster, Wuhan, China); moderate molecular weight protein marker (Thermo Fisher); enhanced chemiluminescence kit (Beyotime, Wuhan, China); fetal bovine serum (GIBCO, US); RPMI-1640 supplemented with phenol red (HYCLONE, US); RIPA buffer and BCA kit (Beyotime, Wuhan, China); 6- and 96-well plates, $25 \mathrm{~cm}^{2}$ plastic culture bottle and 24 well Transwell chamber (BD, US); 
Co170R-230-0200 tri-gas incubator for simulating the culture environment of cells (Eppendorf, Germany); inverted phase contrast microscope (Olympus, Japan); vertical electrophoresis tank and transfer electrophoresis tank (Beijing Liuyi Electronic Co., Ltd).

\section{Methods}

Cell culture: Lung cancer A549 cells were cultured using the RPMI-1640 medium supplemented with $10 \%$ fetal bovine serum, $100 \mathrm{U} / \mathrm{ml}$ penicillin, $100 \mu \mathrm{g} / \mathrm{ml}$ gentamycin and phenol red in an incubator $\left(37^{\circ} \mathrm{C}, 5 \% \mathrm{CO}_{2}\right.$ and saturated humidity), and digested using $0.25 \%$ trypsin for subculture. When the cell infusion reached about $70-80 \%$, cells were starved using the serum-free culture medium followed by $24 \mathrm{~h}$ of culture in an incubator in a low concentration of $\mathrm{O}_{2}$. Thereafter, detections of relevant indicators of A549 cells were carried out. In this experiment, regular condition for culture was set as $37^{\circ} \mathrm{C}, 5 \%$ $\mathrm{CO}_{2}$ and $20 \% \mathrm{O}_{2}$, while the hypoxic condition was set as $1 \%$ $\mathrm{O}_{2}$.

Preparing the growth curve under different culture conditions through CCK-8: Lung cancer A549 cells in $3^{\text {rd }}$ subculture in logarithmic phase were collected for regular digestion, and then seeded on the 96 well plate at density of 1 $\times 10^{4} / \mathrm{ml}(200 \mu \mathrm{L} /$ well $)$, in which we set a well for blank control, where only $200 \mu \mathrm{L}$ medium was added. In this experiment, cells were seeded on two plates. Thereafter, cells were cultured in the hypoxic incubator and regular incubator followed by CCK-8 detections respectively at 3, 6, 12 and $24 \mathrm{~h}$ in 5 experiment wells and 1 blank well, where $20 \mu \mathrm{L} \mathrm{CCK}-8$ was added for $2 \mathrm{~h}$ of incubation. Then, plates were taken out for detection the absorbance (A) at wavelength of $450 \mathrm{~nm}$ with a microplate reader. The growth curve was then prepared with the time (h) as the $\mathrm{x}$-coordinate and $\mathrm{A}$ value as the $\mathrm{y}$ coordinate.

RNA extraction and real-time PCR: Expression of $\mathrm{Wnt} / \beta$ catenin was detected through RT-PCR in following steps: cells in each group were washed using pre-cooled Phosphate Buffer Saline (PBS) three times on ice; in each well, $1 \mathrm{ml}$ TRIzol RNA extraction reagent was added for extracting the total RNA according to the instructions; A260/A280 ratio was also detected via ultraviolet spectrometry to calculate the RNA concentration, which was carried out in triplicate. Afterwards, through reverse transcription of the extracted RNA, cDNA was prepared for following PCR with the primers synthesized by Shanghai Tsingke Biotech Co., Ltd. The primer sequences are shown as follows: $\beta$-actin, forward primer 5'ATGGGGAAGGTGAAGGTCG-3', reverse primer 5'GGGTCATTGATGGCAACAATATC-3'; HIF-1 $\alpha$, forward primer 5'- CGUACUGCACUCCAAAUATT', reverse primer 5'- UAUUUGGAGUGCAGUAGCGTT-3'; $\beta$-catenin forward primer 5'-GCTGCTGTTTTGTTCCGAATGT-3', reverse primer 5'-GCCATTGGCTCTGTTCTGAAGA-3'. Real-time quantitative PCR was carried out in $20 \mu \mathrm{L}$ reaction system supplemented with $10 \mu \mathrm{L}$ mixture, $1 \mu \mathrm{L}$ probe primer and $2 \mu \mathrm{L}$ cDNA, and in following conditions: initial denaturation at $95^{\circ} \mathrm{C}$ for $3 \mathrm{~min}$; 35 cycles of $48^{\circ} \mathrm{C}$ for $30 \mathrm{~s}$ and $72^{\circ} \mathrm{C}$ for $1 \mathrm{~min}$; extension at $72^{\circ} \mathrm{C}$ for $10 \mathrm{~min}$. PCR products were loaded for $1.5 \%$ agarose electrophoresis followed by imaging using gel imager and scan of gray value in triplicate.

Western blotting assay: After regular culture or hypoxic culture for lung cancer A549 cell lines, the adhering cells in 6well plate were washed using cold PBS three times on ice followed by the treatment of RIPA buffer. Cell lysate was then collected using a cell scraper and transferred into a $1.5 \mathrm{ml} \mathrm{EP}$ tube. Following $30 \mathrm{~min}$ of lysis on ice, the lysate was centrifuged at $12000 \mathrm{rpm}$ for $10 \mathrm{~min}$ and the supernatant was preserved at $-20^{\circ} \mathrm{C}$. After the measurement of protein concentration via BCA method, $5 \times$ loading buffer was added into the tube for evenly mixing the proteins that were later placed in $95^{\circ} \mathrm{C}$ water bath for denaturation. The $12 \%$ SDSPAGE was prepared in advance, and in each well, $30 \mu \mathrm{g}$ protein samples were loaded for aggregation of proteins under $80 \mathrm{~V}$. The aggregated proteins were then separated under 100 $\mathrm{V}$ and transferred on a PVDF membrane. After membrane was blocked using 5\% skimmed milk for $1 \mathrm{~h}$, rabbit anti-human polyclonal antibodies of HIF-1 $\alpha$ (1: 800) and $\beta$-catenin (1:1000), and the mouse anti-human monoclonal antibody of $\beta$ actin (1:3000) were added onto the membrane for incubation at $4^{\circ} \mathrm{C}$ overnight. Membrane was then washed three times $(5 \mathrm{~min} /$ time) on a decoloring shaker, and incubated with HRP-labeled goat anti-mouse or anti-rabbit $\mathrm{IgG}$ for $1 \mathrm{~h}$ at room temperature. Membrane was washed three times ( $5 \mathrm{~min} / \mathrm{time})$ again using the Tris-Buffer Saline+Tween 20 (TBST), and treated with ECL reagent for reaction at room temperature for $1 \mathrm{~min}$ followed by color development.

Detecting the invasion capability of cells via Transwell invasion experiment: Before this experiment, all reagent and instruments were placed on ice in advance, and Transwell chambers were placed in the 6-well plate. Fifty $\mu \mathrm{L}$ Matrigel (diluted with serum-free medium, 1:2) was coated on the surface of membrane for $20 \mathrm{~min}$ of incubation at $37^{\circ} \mathrm{C}$. After the Matrigel dried completely, the plate was placed in the incubator for $4 \mathrm{~h}$. The next day, lung cancer A540 cell lines in logarithmic phase were starved in serum-free DMEM for $24 \mathrm{~h}$ followed by digestion with $0.25 \%$ EDTA-trypsin. Sequentially, cell suspension was prepared with the serum-free DMEM, where the cell density was adjusted to $4 \times 10^{5} / \mathrm{ml}$, and the cell viability was above $95 \%$ through trypan blue staining. In the upper chamber, $200 \mu \mathrm{L}$ serum-free cell suspension was added; while in the lower chamber, DMEM supplemented with $10 \%$ fetal bovine serum was added $(600 \mu \mathrm{L} /$ well $)$. Afterwards, chambers were delivered to the incubators for regular culture or hypoxic culture for $24 \mathrm{~h}$. Chambers were then washed three times using PBS to remove the medium, and wet cotton swab was used to collect the cells that failed to pass into the upper chamber. Those cells were fixed in $4 \%$ paraformaldehyde for $20 \mathrm{~min}$. Chambers dried completed at room temperature, stained using crystal violet for $20 \mathrm{~min}$, and delivered under the inverted phase contrast microscope for counting the cells passing through the membrane. Five visions were selected for cell count under the microscope (400X), and the average served as the results. 


\section{Statistical methods}

All data were presented as mean \pm standard deviation, and statistical analysis was performed using SPSS 19.0 for oneway ANOVA. $\mathrm{p}<0.05$ suggested that the difference had statistical significance.

\section{Results}

\section{Hypoxia affects the proliferative ability of lung cancer A549 cell lines}

Lung cancer A549 cells, after $24 \mathrm{~h}$ of regular culture or hypoxic culture, were used to detect the proliferative ability through CCK-8 method, and the results showed that compared with cells in regular environment, the proliferative ability of cells in hypoxic environment was significantly elevated; moreover, the elevation in proliferative ability would be more significant with the extension of time. Differences between the two groups had statistical significance $(\mathrm{p}<0.05$; Figure 1$)$.

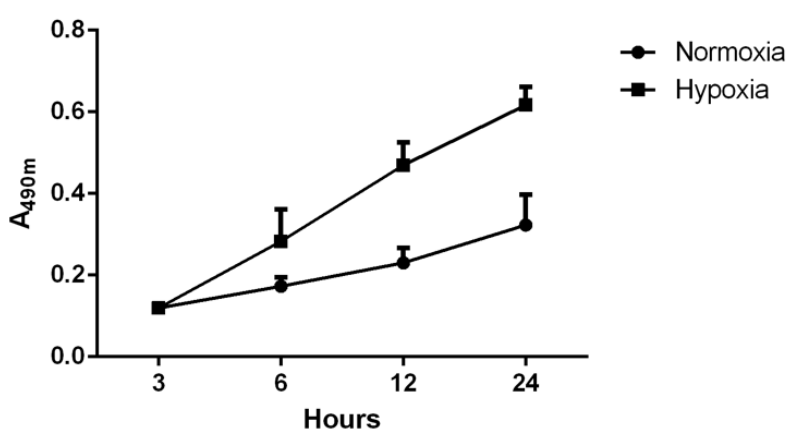

Figure 1. Hypoxia induced A549 cells proliferation.

\section{Hypoxia affects the invasion ability of lung cancer A549 cell lines}

Transwell invasion experiment was carried out to detect the invasion ability of cells after 24 hours of regular culture or hypoxic culture, and the results indicated that the invasion ability of A549 cells was significantly increased in the hypoxic environment in comparison with the regular environment. The difference has statistical significance ( $p<0.05$; Figure 2$)$.

A

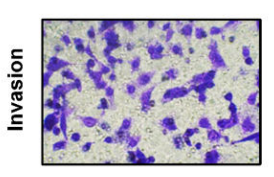

Normoxia
B

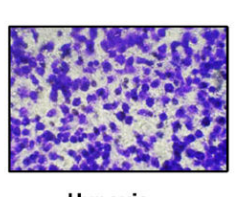

Hypoxia

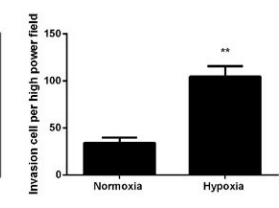

Figure 2. Hypoxia enhances A549 cells invasion (crystal violet staining X200). A. the Transwell invasion assay in A549 cells treated with hypoxia; $B$. the quantitative result of Transwell invasion assay in A549 cells.

\section{Hypoxia affects the Wnt/B-catenin signaling pathway}

After $24 \mathrm{~h}$ of culture under the hypoxic or regular environment, RT-PCR and Western blotting assay were performed to detect the mRNA and protein expressions of $\beta$-catenin. Results showed that compared with the cells in regular environment, mRNA and protein expressions were obviously increased in A549 cells in the hypoxic environment, and the differences had statistical significance $(\mathrm{p}<0.05)$, suggesting that hypoxia can activate the $\mathrm{Wnt} / \beta$-catenin signaling pathway (Figure 3 ).
A

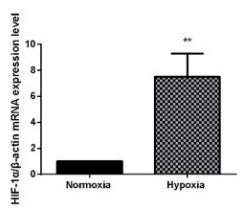

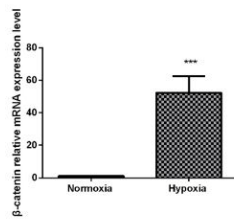

B

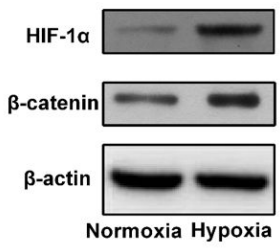

C

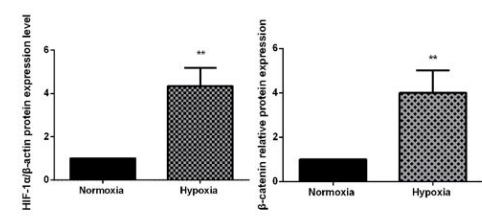

Figure 3. Hypoxia induced $\beta$-catenin signaling activation. A. Western blot assay for $\beta$-catenin protein level in A549 cells.

\section{Silencing $\beta$-catenin affects the $W n t / \beta$-catenin signaling pathway}

After transfection of A549 cells with $\beta$-catenin siRNA and $\beta$ catenin-NC for $48 \mathrm{~h}$, we carried out the RT-PCR and Western blotting assay to measure the mRNA and protein expressions of $\beta$-catenin, and found that compared with the cells in the $\beta$ catenin-NC group and blank control group, a significant decrease was identified in expression of $\beta$-catenin in cells of $\beta$ catenin siRNA group. Differences had statistical significance $(\mathrm{p}<0.05$; Figure 4).
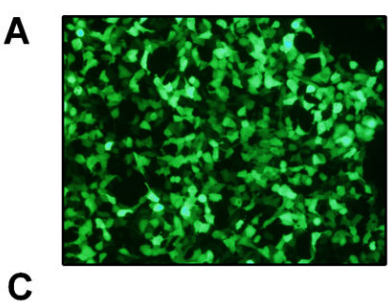

C

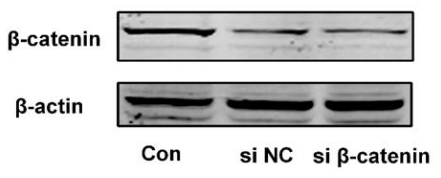

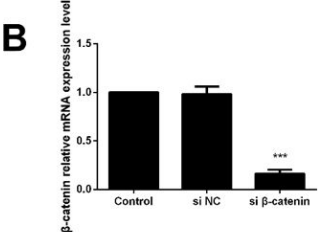

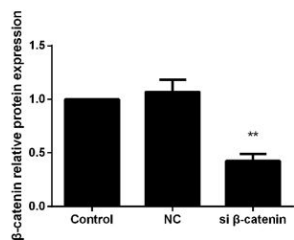

Figure 4. The effective expression of exogenous gene silence of $\beta$ catenin. $A$ and B: RT-PCR analysis of $\beta$-catenin $m R N A$; $C$ : Western blot analysis of $\beta$-catenin protein; D: Quantification of Western blot results. 


\section{The effect of transfection with $\beta$-catenin siRNA on hypoxia-mediated proliferation of $A 549$ cells}

Detections of proliferative ability were performed in the cells cultured in hypoxic or regular environment. The results of CCK-8 method showed that compared with the cells in regular environment, the proliferative ability of A549 cells in hypoxic environment was remarkably enhanced with the extension of time, and the difference had statistical significance $(p<0.05)$; however, after transfection with $\beta$-catenin siRNA, cells experienced a dramatic decrease in proliferative ability with a statistically significant difference $(\mathrm{p}<0.05$; Figure 5$)$.

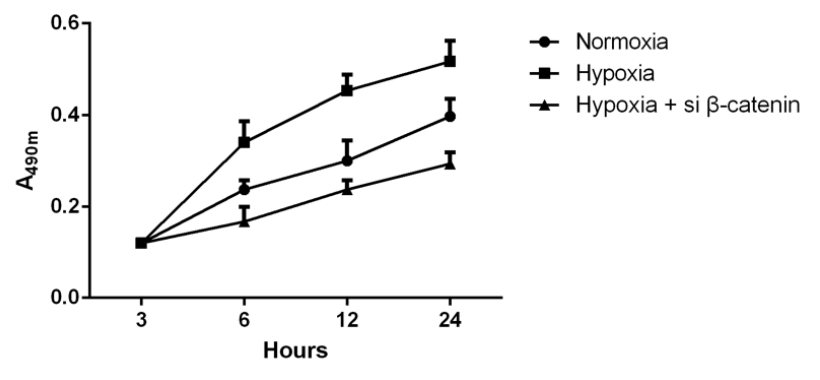

Figure 5. $\beta$-catenin si-RNA inhibits hypoxia induced proliferation.

\section{The effect of transfection with $\beta$-catenin siRNA on hypoxia-mediated proliferation of A549 cells}

In Transwell experiment, we found that after 24 hours of culture in hypoxic environment, the invasion ability of A549 cells in the hypoxic environment was significantly higher than those in the regular environment $(p<0.05)$, and the invasion ability of cells transfected with $\beta$-catenin siRNA was obviously inhibited, in which the differences had statistical significance $(\mathrm{p}<0.05$; Figure 6$)$.

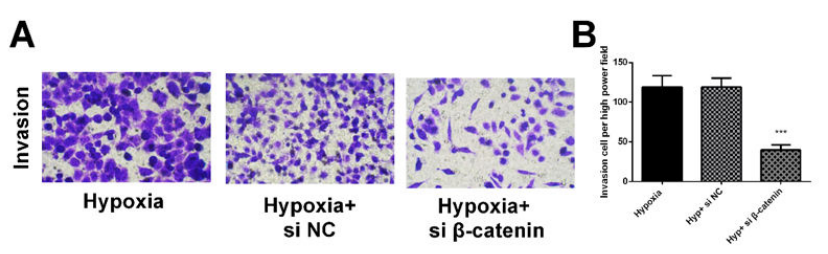

Figure 6. $\beta$-catenin si-RNA attenuates hypoxia induced A549 invasion (crystal violet staining X200). A and B: Transwell assay detect the invasion abilities of $A 549$ and quantification of the results.

\section{Discussion}

At present, lung cancer is one of the malignant tumors severely threatening the health of human beings with the highest morbidity and mortality rate in the world; thus, research to discover the pathogenesis and searching for effective treatment have been one of the hotspots attracting wide attention [14,15]. In healthy people, variations in oxygen concentration show a critical regulatory effect on metabolism, proliferation and differentiation of cells [16]. To adapt to the stress responses to hypoxic metabolism, tumor cells could activate a series of signaling pathways, including the pathways promoting the peripheral angiogenesis, enhancing cell metabolism and migration, or facilitating the abnormal proliferation, metastasis and drug resistance $[17,18]$. Under the hypoxic environment, these biological events could not only alter the biological features of the tumor cells, but also result in the instability in genome of tumor cells, thereby leading to the malignant transformation of tumor, or even metastatic invasion and migration. Lung cancer is one of the most common solid tumors, and during the tumor growth, it is always accompanied by rapid abnormal proliferation, which will result in the insufficiency of local blood supply, and furthermore, the ischemic and hypoxic microenvironment, where survived tumor cells are inevitably exacerbate the hypoxia in the microenvironment. Under the hypoxic and ischemic condition, tumor cells can alter the surrounding environment through autocrine or paracrine, thereby accelerating the growth and progression of tumor. This process is also frequently accompanied with some variations of their own features, e.g. stronger local infiltrative and distant invasive ability, which are conducive to the metastasis of tumor cells.

Wnt/ $\beta$-catenin signaling pathway is widely involved in the cellular information regulation, and plays vital roles in cell proliferation, tissue repair, embryonic development and development and progression of tumors, including lung cancer [19]. $\beta$-catenin, an intracellular multifunctional protein and the core regulatory element in Wnt signal pathway, mainly participates in regulation of intercellular adhesion. Normally, in normal, mature tissue cells, $\beta$-catenin in cytoplasm can bind with the E-cadherin on cell membrane to promote the intercellular adhesion, but downstream target genes can hardly be activated due to the insufficiency of free $\beta$-catenin in cytoplasm. When Wnt signal pathway is activated by the stimuli to cells, Wnt ligand, binding with the Frizzled/LRP receptor, can activate the Dsh through phosphorylation, thereby isolating the $\beta$-catenin from $\mathrm{APC} / \mathrm{Ax}^{-}$in/GSK-3 $\beta$; the accumulation of $\beta$-catenin would further enter the nuclear to combine with the TCF/LEF complex, thereby activating the transcription of downstream target genes, through which Wnt signal pathway can regulate various biological events, like cell proliferation, apoptosis, mobility and differentiation [20,21]. Research has reported the close correlations of $\beta$-catenin with the development and progression of a variety of tumors, including liver cancer [22], gastric cancer [23] and lung cancer [24]. All these studies have shown that $\beta$-catenin is highly expressed in the above tumor tissues. In liver cancer cells, Wnt $/ \beta$-catenin signaling pathway can promote the invasion and metastasis of cells through enhancing the hypoxia-induced epithelial-mesenchymal transition, and the expression of $\beta$ catenin is negatively correlated with the survival duration of patients after resection $[25,26]$. Also, in colorectal cancer cells, hypoxic environment can facilitate cell growth and invasion through activation of Nur77- $\beta$-catenin loop [27]. Nevertheless, there remain no literatures reporting the mechanism of $\mathrm{Wnt} / \beta$ catenin signaling pathway in regulation of proliferation and invasion of lung cancer cells in hypoxic microenvironment. 
In this study, we firstly investigated the effect of in-vitro hypoxic microenvironment on proliferation and invasion abilities of A549 cells, and the results showed that compared with cells in regular environment, the proliferative ability of cells in hypoxic environment was significantly elevated; moreover, the elevation in proliferative ability would be more significant with the extension of time. Sequentially, we studied the molecular mechanism leading to the previous result in the hypoxic microenvironment, and found that after $24 \mathrm{~h}$ of culture under hypoxic environment, $\beta$-catenin signal pathway in A549 cells was significantly activated, suggesting that it might be involved in the abnormal proliferation and invasion of lung cancer cells in hypoxic environment. To further clarify the mechanism of Wnt/ $\beta$-catenin signaling pathway, we blocked the expression via transfection with $\beta$-catenin siRNA, and further detected the effect on proliferation and invasion of cells; results showed that after silencing the $\mathrm{Wnt} / \beta$-catenin signaling pathway, hypoxia-induced proliferation and invasion of lung cancer cells were blocked, indicating the critical role of $\mathrm{Wnt} / \beta$-catenin signaling pathway in this process. Thus, we inferred that through activation of $\mathrm{Wnt} / \beta$-catenin signaling pathway, the transcription of downstream target gene could be further activated for promoting the abnormal proliferation and distant invasion and metastasis of lung cancer cells, thereby facilitating the development and progression of lung cancer.

In conclusion, we, in this study, preliminarily confirmed that in A549 cell lines, hypoxic microenvironment can promote the abnormal proliferation and distant metastasis and invasion of tumor cells through $\mathrm{Wnt} / \beta$-catenin signaling pathway, which is conducive to deepening the understanding of the pathogenesis of lung cancer. Thus, in-depth research on the roles and mechanism of $\mathrm{Wnt} / \beta$-catenin signaling pathway in development and progression of lung cancer, and the techniques for blocking the relevant targets of $\mathrm{Wnt} / \beta$-catenin signaling pathway to inhibit the abnormal proliferation and distant metastasis and invasion of tumor cells can provide new ideas and evidence for individualized treatment of lung cancer with a wide clinical significance and a promising application prospect.

\section{References}

1. Hong QY, Wu GM, Qian GS, Hu CP, Zhou JY. Prevention and management of lung cancer in China. Cancer 2015; 121: 3080-3088.

2. Pastorkova Z, Skarda J, Andel J. The role of microRNA in metastatic processes of non-small cell lung carcinoma. Biomedical papers of the Medical Faculty of the University Palacky, Olomouc, Czechoslovakia 2016; 160: 343-357.

3. Kumarakulasinghe NB, Van Zanwijk N, Soo RA. Molecular targeted therapy in the treatment of advanced stage non-small cell lung cancer (NSCLC). Respirology (Carlton, Vic) 2015; 20: 370-378.

4. Rafei H, El-Bahesh E, Finianos A, Nassereddine S, Tabbara I. Immune-based therapies for non-small cell lung cancer. Anticancer Res 2017; 37: 377-387.
5. Durm G, Hanna N. Second-line chemotherapy and beyond for non-small cell lung cancer. Hematol Oncol Clin N Am 2017; 31: 71-81.

6. Parks SK, Cormerais Y, Marchiq I. Hypoxia optimises tumour growth by controlling nutrient import and acidic metabolite export. Mol Aspec Med 2016; 47: 3-14.

7. Harris BH, Barberis A, West CM. Gene Expression signatures as biomarkers of tumour hypoxia. Clin Oncol Royal Coll Radiol Great Britain 2015; 27: 547-560.

8. Gilkes DM, Semenza GL, Wirtz D. Hypoxia and the extracellular matrix: drivers of tumour metastasis. Nat Rev Cancer 2014; 14: 430-439.

9. Hubbi ME, Semenza GL. Regulation of cell proliferation by hypoxia-inducible factors. Am J Cell Physiol 2015; 309: 775-782.

10. Madanecki P, Kapoor N, Bebok Z, Ochocka R, Collawn JF. Regulation of angiogenesis by hypoxia: the role of microRNA. Cell Mol Biol Lett 2013; 18: 47-57.

11. Yuen A, Diaz B. The impact of hypoxia in pancreatic cancer invasion and metastasis. Hypoxia (Auckland, NZ) 2014; 2: 91-106.

12. Vilchez V, Turcios L, Marti F. Targeting Wnt/beta-catenin pathway in hepatocellular carcinoma treatment. World J Gastroenterol 2016; 22: 823-832.

13. Duan C, Li T, Liu L. Efficacy of limited fluid resuscitation in patients with hemorrhagic shock: a meta-analysis. Int $\mathrm{J}$ Clin Exp Med 2015; 8: 11645-11656.

14. Yang J, Chen J, He J. Wnt signaling as potential therapeutic target in lung cancer. Exp Opin Ther Targ 2016; 20: 999-1015.

15. Sihoe AD, Van Schil P. Non-small cell lung cancer: when to offer sublobar resection. Lung Cancer (Amsterdam, Netherlands) 2014; 86: 115-120.

16. Eskandani M, Vandghanooni S, Barar J. Cell physiology regulation by hypoxia inducible factor-1: targeting oxygenrelated nanomachineries of hypoxic cells. Int $\mathrm{J}$ Biol Macromol 2017; 99: 46-62.

17. Marchiq I, Pouyssegur J. Hypoxia, cancer metabolism and the therapeutic benefit of targeting lactate/ $\mathrm{H} \quad(+)$ symporters. J Mol Med (Berlin, Germany) 2016; 94: 155-171.

18. Manoochehri Khoshinani H, Afshar S, Najafi R. Hypoxia: a double-edged sword in cancer therapy. Cancer Invest 2016; 34: 536-545

19. Yao H, Ashihara E, Maekawa T. Targeting the Wnt/ $\beta$ catenin signaling pathway in human cancers. Expert Opin Ther Targets 2011; 15: 873-887.

20. Duan C, Chen K, Yang G.HIF-1a regulates Cx40dependent vasodilatation following hemorrhagic shock in rats. Am J Transl Res 2017; 9: 1277-1286.

21. Guo Y, Xiao L, Sun L. Wnt/beta-catenin signaling: a promising new target for fibrosis diseases. Physiol Res 2012; 61: 337-346.

22. Monga SP. Role of Wnt/beta-catenin signaling in liver metabolism and cancer. Int J Biochem Cell Biol 2011; 43: 1021-1029. 
23. Cai J, Feng D, Hu L. FAT4 functions as a tumour suppressor in gastric cancer by modulating Wnt/betacatenin signalling. Br J Cancer 2015; 113: 1720-1729.

24. Chen X, Song X, Yue W, Chen D. Fibulin-5 inhibits Wnt/ $\beta$ catenin signaling in lung cancer. Oncotarget 2015; 6: 15022-15034.

25.Zhang Q, Bai X, Chen W. Wnt/beta-catenin signaling enhances hypoxia-induced epithelial-mesenchymal transition in hepatocellular carcinoma via crosstalk with hif-1alpha signaling. Carcinogene 2013; 34: 962-973.

26. Liu L, Zhu XD, Wang WQ. Activation of beta-catenin by hypoxia in hepatocellular carcinoma contributes to enhanced metastatic potential and poor prognosis. Clinic Off J Am Assoc Cancer Res 2010; 16: 2740-2750.
27. To SK, Zeng WJ, Zeng JZ. Hypoxia triggers a Nur77-betacatenin feed-forward loop to promote the invasive growth of colon cancer cells. Br J Cancer 2014; 110: 935-945.

\section{*Correspondence to}

Xiaofei Zeng

Department of Cardiothoracic Surgery

The First Affiliated Hospital of Chengdu Medical College

PR China 